\title{
ON TOPOLOGISING THE FIELD $C(t)$
}

\section{J. H. WILLIAMSON}

In many questions concerning the existence of fields over the complex field, with topologies satisfying given compatibility axioms, it is sufficient to restrict attention to the field $C(t)$ of rational functions of the indeterminate $t$ over the complex field $C$. Every division algebra over $C$, other than $C$ itself, contains a subfield isomorphic to $C(t)$. It will be shown below that $C(t)$ can be topologised so that addition and multiplication are continuous operations; this settles a question raised by Kaplansky [2, p. 811]. If local convexity is not required, there is already a well known metrisable topology satisfying the requirements. It is also possible to produce a locally convex metrisable topology with the required properties. If we postulate a topology compatible with the linear space structure of $C(t)$, then it will be shown that if multiplication is to be continuous, the topology must not be too coarse. It will be shown also that it must not be too fine, and that, in fact, in the finest locally convex linear space topology on $C(t)$, multiplication is not continuous.

1. We consider first the classical theorem usually associated with the names of Mazur and Gelfand; in a generalised form, due to Arens [1, Theorem 1], this is: Let $A$ be a division algebra over $C$, with a topology such that (i) there is a total set of continuous linear functionals; (ii) addition and scalar multiplication are continuous; (iii) multiplication by an element of $A$ is continuous; (iv) inversion is continuous. Then $A=C$. The conditions of the theorem can be relaxed in various ways, the proof involving Liouville's theorem remaining valid. The following extension is comparatively trivial:

Theorem 1. Let $A$ be a division algebra over $C$, with a topology such that

(i') there is one nonzero continuous linear functional;

(ii) addition and scalar multiplication are continuous;

(iii) multiplication (left or right) by an element of $A$ is a continuous operation;

(iv') for each complex number $\lambda_{0}$ there is a non-negative integer $n\left(\lambda_{0}\right)$ such that $\left(\lambda-\lambda_{0}\right)^{n}\left\{\left(t-\lambda_{0} e\right)^{-1}-(t-\lambda e)^{-1}\right\}$ is bounded for all $\lambda$ sufficiently near $\lambda_{0}$; and there is a non-negative integer $n^{\prime}$ such that

Presented to the Society, February 27, 1954; received by the editors December 8 , 1953. 
$\lambda^{-n^{\prime}}(t-\lambda e)^{-1}$ is bounded for all sufficiently large $|\lambda|$. (e denotes the unit of $A$.)

Then $A=C$.

Proof. The condition ( $i^{\prime}$ ) is easily seen to imply (i), given (iii) and the fact that $A$ is a division algebra. Condition (iv') implies (in view of the relation

$$
\begin{aligned}
(t-\lambda e)^{-1}= & \sum_{r=0}^{n}\left(\lambda-\lambda_{0}\right)^{r}\left(t-\lambda_{0} e\right)^{-r-1} \\
& +\left(\lambda-\lambda_{0}\right)^{n+1}(t-\lambda e)^{-1}\left(t-\lambda_{0} e\right)^{-n-1}
\end{aligned}
$$

and the corresponding formula for large $|\lambda|)$ that if $t$ is not a scalar multiple of $e$ and $u$ is any continuous linear functional, then $u\left((t-\lambda e)^{-1}\right)$ is a bounded analytic function of $\lambda$. The standard arguments can thus be used to give the required result.

Corollary. If $A$ has a norm such that (iii) holds in the associated topology, then $A=C$.

Proof. It is clear that (i') and (ii) are satisfied. Since

$$
(t-\lambda e)^{-1}=\left(t-\lambda_{0} e\right)^{-1}+\left(\lambda-\lambda_{0}\right)\left(t-\lambda_{0} e\right)^{-1}(t-\lambda e)^{-1},
$$

and multiplication by $\left(t-\lambda_{0} e\right)^{-1}$ is continuous, it follows that

$$
\left\|(t-\lambda e)^{-1}\right\| \leqq\left\|\left(t-\lambda_{0} e\right)^{-1}\right\|+k\left|\lambda-\lambda_{0}\right|\left\|(t-\lambda e)^{-1}\right\|,
$$

whence $(t-\lambda e)^{-1}$ is bounded in a neighbourhood of $\lambda_{0}$. It can be shown similarly that $(t-\lambda e)^{-1} \rightarrow 0$ as $|\lambda| \rightarrow \infty$; so condition (iv') of the theorem is satisfied, with $n\left(\lambda_{0}\right)=0, n^{\prime}=0$.

In proving the corollary, no use has been made of completeness in any way. As proved by Gelfand, separate continuity of multiplication implies $\left\|t_{1} t_{2}\right\| \leqq\left\|t_{1}\right\|\left\|t_{2}\right\|$, in essence; the proof rests on completeness, and hence on a category argument. Arens [1] has shown that if there is a norm with $\left\|t_{1} t_{2}\right\| \leqq\left\|t_{1}\right\|\left\|t_{2}\right\|$, then inversion is continuous. So the result of the corollary is known, although the present method of proof appears to be new.

The above conditions (i)-(iv) or $\left(\mathrm{i}^{\prime}\right)-\left(\mathrm{iv}^{\prime}\right)$ can turn up in various disguises. For instance: let $A$ be a division algebra over $C$, containing a subset $B$ such that (a) $B \neq A, B \neq\{0\}$; (b) for each $x \in A$, there is a nonzero scalar $\lambda$ such that $\lambda x \in B$; (c) if $x \in B$, and $|\alpha| \leqq 1$, then $\alpha x \in B$; if $x, y \in B$ then $(x+y) / 2 \in B$; (d) for each $x \in A$ there is a scalar $\lambda$ such that $x B \subset \lambda B$. Then $A=C$. For, the function $N(x)$ $=$ inf $|\alpha|$ for $x \in \alpha B$ will then be a norm on $A$ such that (iii) holds, and the corollary will be applicable. 
In passing, it may be mentioned that a topological division algebra of Kaplansky's "type V" [3, p. 910] over $C$ must coincide with $C$; for the condition that the set $B^{-1}$ is bounded whenever $B$ is disjoint from a neighbourhood of 0 implies condition (iv') of Theorem 1 , with $n=n^{\prime}=0$.

2. We now show that it is possible to introduce a metrisable topology in $C(t)$ such that addition, multiplication, and inversion are continuous. Consider the space $M(0,1)$ of functions which are almost everywhere finite and Lebesgue measurable in the interval $(0,1)$, with the topology of convergence in measure in $(0,1)$. Explicitly, the basic neighbourhoods of 0 will be the sets

$$
N(k, \epsilon)=\{f: m\{t:|f(t)| \geqq k\}<\epsilon\}
$$

where $m$ is Lebesgue measure on subsets of $(0,1)$, and $k$ and $\epsilon$ are arbitrary positive real numbers. In this topology, addition is continuous (at $(0,0)$, hence everywhere); given $N(k, \epsilon)$ we have $N(k / 2, \epsilon / 2)+N(k / 2, \epsilon / 2) \subset N(k, \epsilon)$. Multiplication by an element of $M(0,1)$ is continuous (at 0 , hence everywhere); given $f$ and $N(k, \epsilon)$, choose $k^{\prime}$ so that $m\left\{t:|f(t)| \geqq k^{\prime}\right\}<\epsilon / 2$, and then $f N\left(k / k^{\prime}, \epsilon / 2\right)$ $\subset N(k, \epsilon)$. Multiplication is continuous at $(0,0)$; given $N(k, \epsilon)$ we have $N\left(k^{1 / 2}, \epsilon / 2\right) N\left(k^{1 / 2}, \epsilon / 2\right) \subset N(k, \epsilon)$, and hence, in view of the previous remarks, multiplication is continuous everywhere. In particular, scalar multiplication is continuous. Finally, inversion is continuous; given $N(k, \epsilon)$ we have $f^{-1} \in 1+N(k, \epsilon)$ whenever $f \in 1$ $+N\left(k^{\prime}, \epsilon\right)$, where $k^{\prime}=\inf \{k / 2,1 / 2\}$, and $f^{-1}$ exists.

Now regard $t$ as a real variable, and consider the topology induced on $C(t)$ as a subset of $M(0,1)$. The continuity of the algebraic operations is unaffected; and the topology on $C(t)$ is metrisable, since it is Hausdorff and there is a countable basic set of neighbourhoods.

There can be no nonzero linear functionals on $C(t)$ which are continuous in the above topology; for if there were one such functional, we would have $C(t)=C$, by Theorem 1 . It is easy to deduce from this that there are no nonzero linear functionals on $M(0,1)$ which are continuous in the topology of convergence in measure. For, the set of rational functions of $t$ (in fact, the set of polynomials in $t$ ) is dense (in measure) in $M(0,1)$.

3. We now produce a locally convex metrisable topology on $C(t)$ in which addition and multiplication are continuous-in view of the convexity, addition is automatically continuous, so that we shall have only the continuity of multiplication to consider. Let us define, for each positive integer $n$, a sequence $w(n)=\left\{\cdots, w_{-r}, \cdots\right.$, $\left.w_{-1}, w_{0}, w_{1}, \cdots, w_{s}, \cdots\right\}$, where 


$$
\begin{array}{rlrl}
w_{-r} & =(r+1)^{n(r+1)} & & \text { if } r \geqq 1 ; \\
w_{0} & =1 ; & & \\
w_{s}=(s+1)^{-(s+1) / n} & & \text { if } s \geqq 1 .
\end{array}
$$

The set of all such sequences will be denoted by $S$.

Lemma 1. If $u \in S$, there exists $v \in S$ such that $v_{r} v_{s} \geqq u_{r+s}$ for all integers $r$, s. In fact, if $u=w(n)$, we can take $v=w(4 n)$.

Proof. The verification of this is rather tedious, but quite straightforward if the various cases which may arise (according to the signs of $r, s$, and $r+s)$ are treated separately.

Next, let $r(t)$ be any rational function of $t$, with Laurent expansion at $t=0, r(t)=\sum a_{s} t^{s}$. For any $u \in S$, the series $\sum_{s=-\infty}^{\infty}\left|a_{8}\right| u_{s}$ is clearly convergent. Consider the subsets of $C(t)$ :

$$
N(u, \epsilon)=\left\{r(t): \sum\left|a_{s}\right| u_{s}<\epsilon\right\}
$$

as $u$ varies over all sequences of $S$, and $\epsilon$ over all positive real numbers. That these sets can be taken as a basic set of neighbourhoods of 0 in a locally convex Hausdorff linear space topology on $C(t)$ is immediate. Multiplication by an element of $C(t)$ is continuous (at 0 , and hence everywhere); given $r(t)=\sum a_{s} t^{s}$ and $N(u, \epsilon)$, choose $v$ as in Lemma 1 . Then $\sum\left|a_{s}\right| v_{s}$ ( $=k$, say) is finite, and it is easy to verify that $r N(v, \boldsymbol{\epsilon} / k) \subset N(u, \epsilon)$. Multiplication is continuous at $(0,0) ; v$ being as above we have $N\left(v, \epsilon^{1 / 2}\right) N\left(v, \epsilon^{1 / 2}\right) \subset N(u, \epsilon)$. Hence multiplication is continuous everywhere. Since there is a countable set of basic neighbourhoods (the set $S$ being countable) the topology is metrisable.

$C(t)$ is not complete in the above topology; the Cauchy sequence of rational functions $r_{n}(t)=\sum_{s=1}^{n}(s+1)^{-s(s+1)} t^{-s}$ has no limit in $C(t)$.

4. If in two topologies on $C(t)$ addition and multiplication are continuous, the same is true of their union; if both are locally convex, so is their union. It follows that there is on $C(t)$ a unique finest Hausdorff topology in which addition and multiplication are continuous; and a unique finest locally convex Hausdorff topology satisfying the same conditions.

Assuming a topology compatible with the linear space structure of $C(t)$, it is clear that if multiplication is to be continuous then the topology must not be too coarse. In fact, every neighbourhood (of 0 ) must contain a neighbourhood $N$ such that sup $|\alpha|$, for $\alpha r(t) \in N$, is finite for each $r(t) \in C(t)$. (In the locally convex case, this means that there is a basic set of neighbourhoods defined by a family of norms.) For, let $N_{0}$ be a given neighbourhood, other than the whole space 
$C(t)$; let $r_{0}(t) \notin N_{0}$. There are neighbourhoods $N_{1}$ and $N_{2}$ such that $N_{1} N_{2} \subset N_{0}$. Suppose that sup $|\alpha|$ for $\alpha r_{1}(t) \in N_{1}$ is not finite; we can then find $\alpha^{\prime}$ and $\beta^{\prime}$ such that $\alpha^{\prime} r_{1}(t) \in N_{1}, \beta^{\prime}\left\{r_{1}(t)\right\}^{-1} r_{0}(t) \in N_{2}$, and $\alpha^{\prime} \beta^{\prime}=1$. Then $r_{0}(t) \in N_{0}$, contrary to assumption; hence sup $|\alpha|$ for $\alpha r(t) \in N_{1}$ is finite for each $r(t) \in C(t)$. $N_{0}$ clearly contains a nonzero scalar multiple of $N_{1}$, which establishes our assertion.

On the other hand, if multiplication is to be continuous, the topology must not be too fine. We shall show that if $C(t)$ is given the finest topology compatible with its linear space structure, multiplication is not continuous. Thus the problem arises of identifying explicitly the two (possibly equivalent) unique finest Hausdorff linear space topologies, in which multiplication is continuous. We do not attempt to discuss this problem here.

It may be remarked that in the finest linear space topology on $C(t)$, multiplication by a rational function is always continuous, since if the collection $\left\{N_{i}\right\}$ of subsets of $C(t)$ can be taken as a basic set of neighbourhoods of 0 in some linear space topology, so can the collection $\left\{r_{j} N_{i}\right\}$ as $r_{j}$ runs through all nonzero rational functions, and $N_{i}$ the given collection. The same remark applies with the additional qualification "locally convex."

Now, every rational function can be expressed uniquely as a finite sum

$$
f(t)=\sum_{r \geqq 0} b_{\infty, r} t^{r}+\sum_{\lambda} \sum_{r \geqq 1} b_{\lambda, r}(t-\lambda)^{-r} .
$$

Every linear functional on $C(t)$ is of the form

$$
u(f)=\sum_{r \geqq 0} b_{\infty, r} u_{\infty, r}+\sum_{\lambda} \sum_{r \geqq 1} b_{\lambda, r} u_{\lambda, r}
$$

A set $B$ of linear functionals on $C(t)$ is bounded if, for each rational function $f, \sup _{u \in B}|u(f)|$ is finite. The polar set $B^{0}$ of a bounded set $B$ of linear functionals will be a neighbourhood of 0 in the finest (locally convex) linear space topology on $C(t)$. Thus in particular there is a neighbourhood $N$ such that $\left|b_{\lambda, 1}\right| \leqq 1$ for all functions in $N$ and all $\lambda$; it is sufficient to take for $N$ the set $B^{0}$, where $B$ is the set consisting of all functionals $u$ with $u_{\lambda, 1}=1$ for some $\lambda$, and all other coordinates zero.

The next lemma is stated in greater generality than is actually required; we need the result only for real-valued functions of a complex variable.

Lemma 2. Let $\phi(z)$ be a real- (or complex-) valued function on a complete metric space, such that, for each $z, \phi(z) \neq 0$. Let $X$ be the set of points $z_{0}$ such that $\phi(z) \rightarrow 0$ as $z \rightarrow z_{0}$. Then int $\bar{X}$ is empty (that is, 
$X$ is an "ensemble rare").

Proof. Suppose not. Then there will be a sphere $d\left(z, z_{0}\right)<\epsilon_{0}$ in which $X$ is dense; let $z_{1} \in X$ be such that $d\left(z_{0}, z_{1}\right)<\epsilon_{0} / 2$. Choose $\epsilon_{1}<\epsilon_{0} / 2$ such that $|\phi(z)|<1$ for $d\left(z, z_{1}\right)<\epsilon_{1}$; let $z_{2} \in X$ be such that $d\left(z_{1}, z_{2}\right)<\epsilon_{1} / 2$. Choose $\epsilon_{2}<\epsilon_{1} / 2$ so that $|\phi(z)|<1 / 2$ for $d\left(z, z_{2}\right)<\epsilon_{2}$; let $z_{3} \in X$ be such that $d\left(z_{2}, z_{3}\right)<\epsilon_{2} / 2 \ldots$. Continuing in this way, we obtain a convergent sequence of points, $z_{0}, z_{1}, z_{2}, \cdots$, with a limit $z^{\prime}$, say; we have $\left|\phi\left(z^{\prime}\right)\right|<1 / n$ for all $n$, so that $\phi\left(z^{\prime}\right)=0$, contrary to assumption.

COROLlary. Let $Y$ be the set of points $z$ with the following property: there exists a positive real number $\delta(z)$ such that, for every $\epsilon>0$, there is a point $z^{*} \neq z$ with $d\left(z, z^{*}\right)<\epsilon$ and $\left|\phi\left(z^{*}\right)\right| \geqq \delta(z)$. Then $Y$ is dense.

For clearly $Y$ and $X$ are complementary.

THEOREM 2. In the finest (locally convex) linear space topology on $C(t)$, multiplication is not continuous.

Proof. Assume that multiplication is continuous. Let $N$ be a neighbourhood of 0 such that, for all rational functions in $N,\left|b_{\lambda, 1}\right| \leqq 1$ for all $\lambda$. There will be a neighbourhood $N^{\prime}$ such that $N^{\prime} N^{\prime} \subset N$; let $\phi(\lambda)=\sup |\alpha|$ for $\alpha(t-\lambda)^{-1} \in N^{\prime}$. Then $\phi(\lambda)$ is never zero; hence, by Lemma 2, corollary, there will be a point $\lambda_{0}$ and a $\delta>0$ such that, given $\epsilon>0$, there is a point $\lambda \neq \lambda_{0}$, with $\left|\lambda-\lambda_{0}\right|<\epsilon$ and $|\phi(\lambda)| \geqq \delta$. We have

$$
2^{-1} \phi\left(\lambda_{0}\right) \phi(\lambda)\left(t-\lambda_{0}\right)^{-1}(t-\lambda)^{-1} \in N^{\prime} N^{\prime} \quad \text { for all } \lambda \text {; }
$$

that is,

$$
2^{-1} \phi\left(\lambda_{0}\right) \phi(\lambda)\left(\lambda_{0}-\lambda\right)^{-1}\left\{\left(t-\lambda_{0}\right)^{-1}-(t-\lambda)^{-1}\right\} \in N .
$$

But, for this function, the coefficient $b_{\lambda, 1}$ is clearly unbounded. We have thus reached a contradiction, and the theorem is proved.

In fact the above proof shows that multiplication is not continuous in any linear space topology in which $B^{0}$ (as defined above) is a neighbourhood of 0 .

\section{REFERENCES}

1. R. Arens, Bull. Amer. Math. Soc. vol. 53 (1947) pp. 623-630.

2. I. Kaplansky, Bull. Amer. Math. Soc. vol. 54 (1948) pp. 809-826.

3. ——, Bull. Amer. Math. Soc. vol. 54 (1948) pp. 909-916.

Queen's University, Belfast 